\title{
La chefferie autrement. Une notion mille-feuilles bonne à repenser
}

A Different Chiefdom. A Multi-Layered Notion "Good to re-Think"

\section{Viviane Cretton}

\section{(2) OpenEdition \\ Journals}

Édition électronique

URL : http://journals.openedition.org/jso/7425

DOI : $10.4000 /$ jso. 7425

ISSN : $1760-7256$

Éditeur

Société des océanistes

\section{Édition imprimée}

Date de publication : 15 décembre 2015

Pagination : 267-282

ISBN : 978-2-85430-126-7

ISSN : 0300-953x

\section{Référence électronique}

Viviane Cretton, "La chefferie autrement. Une notion mille-feuilles bonne à repenser », Journal de la Société des Océanistes [En ligne], 141 | juillet-décembre 2015, mis en ligne le 15 décembre 2018, consulté le 02 mai 2019. URL : http://journals.openedition.org/jso/7425; DOI : 10.4000/jso.7425 


\section{La chefferie autrement. Une notion mille-feuille bonne à repenser}

par

Viviane CRETTON*

\section{RÉSUMÉ}

Cet article présente la réalité polyphonique, historiquement stratifiée et située de la notion de chefferie dans le contexte de la politique fidjienne récente. Sur la base d'une enquête de terrain de longue durée, il explore la complexité et le pluralisme inhérents à la figure du chef à Fidji. La notion de chefferie est d'abord déconstruite au prisme des influences occidentales et coloniales qui l'ont façonnée. L'hétérogénéité qui a caractérisé l'ensemble des chefs fidjiens durant le coup d'État de 2000 est ici mise en évidence. Puis le texte revisite le rôle de médiation joué par les leaders légitimes (et moins légitimes) durant ce coup d'Etat. Il examine l'efficacité de certaines pratiques spécifiquement fidjiennes de résolution du conflit qui mettent en jeu des objets de chef (dent de cachalot ou tabua). En continuité, l'article relie les trajectoires de plusieurs dirigeants successifs après l'indépendance à diverses interprétations, également plurielles, de leur mana. Ce faisant, il analyse la nouvelle configuration de la classe sociopolitique fidjienne dans toute sa complexité.

Mots-CLÉs : chef, tabua, mana, coup d'État, anthropologie politique

«Ce que vous appelez démocratie n'est pas quelque chose de nouveau pour nous. C'est notre façon de vivre au village. » (Adi Litia Cakobau, vice-présidente du grand conseil des chefs, Suva, 21 mars 2000)

Emblème de la chefferie à Fidji, le grand conseil des chefs a été abrogé en mars 2012. En conséquence du coup d'État orchestré le 5 décembre 2006 par Voreqe Bainimarama, actuel Premier ministre alors

\begin{abstract}
This paper ethnographies the highly stratified and situated notion of chieftainship within recent Fijian politics. Based on a long term fieldwork, the article explores the complexity and the pluralism that are inherent in chief figure in Fiji. A first part deconstructs the notion on chieftainship in Fiji through the prism of western and colonial influences. During the 2000 coup d'Etat, heterogeneity appeared to be the main feature of the Fijian chiefs as a whole. A second part revisits the mediation enacted by the legitimate and less legitimate leaders during the 2000 Coup d'Etat. It examines the efficiency of some distinctively Fijian practices involving chiefly artefacts (sperm whale's tooth or tabua) to resolve conflict. In the end, the paper connects the trajectory of successive post-independance leaders with diverse understandings of their mana. This brings to enlighten the configuration of the new socio-political class in Fiji in all of its complexity.
\end{abstract}

KEYwords: chief, tabua, mana, coup d'État, anthropologie politique

amiral, ses séances étaient déjà suspendues depuis 2007. Communément considéré par les habitants de Fidji et par les anthropologues comme l'instance traditionnelle suprême, le grand conseil des chefs a bénéficié depuis l'indépendance en 1970 d'un droit de veto sur les décisions prises dans le cadre du Parlement. Souvent défini comme le garant des droits coutumiers des Fidjiens, ce conseil avait été formalisé par l'administration coloniale de Sir

* University of Applied Sciences and Arts, Western Switzerland, viviane.cretton@hevs.ch 
Arthur Hamilton Gordon, dès 1875, dans l'objectif de faciliter l'administration de la colonie (18741970) et de sélectionner des représentants fidjiens au gouvernement colonial (Norton, $2000: 145$ ).

L'abrogation planifiée du grand conseil des chefs met fin à la trajectoire d'une institution fidjienne aussi valorisée au plan symbolique que controversée au niveau politique. Ce changement invite à repenser la chefferie à rebours du sens commun. Comprise comme un système stratifié de pouvoirs anciens et nouveaux, symboliques et politiques, elle s'incarne dans la figure du chef, à différentes échelles (famille, village, district, nation) et selon diverses conceptions (emic, etic). À Fidji comme ailleurs dans le Pacifique, la signification exacte du terme " chef » diffère d'une communauté à l'autre. Dans l'usage des auteurs en général, il désigne un leader politique qui dessine son autorité et son influence à partir d'un discours issu de la tradition locale (White et Lamont, 1997 : 10).

Sur la base d'une immersion ethnographique de longue durée $(1997$; 2000-2001 ; 2002 ; 2014), cet article propose de revisiter le rôle des chefs et celui d'autres leaders légitimes et moins légitimes durant le coup d'État de 2000. En mettant en évidence l'hétérogénéité qui caractérise les chefs coutumiers fidjiens dans leur ensemble, hier comme aujourd'hui, il offre de jeter un nouvel éclairage sur la situation actuelle, esquissant les récurrences et retournements conjoncturels qui la façonnent.

La première partie s'attachera à déconstruire la notion de chefferie à Fidji, au prisme des influences occidentales et coloniales qui l'ont façonnée. Elle fera émerger le pluralisme inhérent à la notion de chefferie, tel qu'il s'est révélé durant le coup d'État de 2000. La deuxième partie sera consacrée, d'une part, à l'efficacité des transactions symboliques qui ont contribué à la résolution du conflit en 2000. Les pratiques spécifiquement fidjiennes qui se sont déployées au cœur de la crise politique mettent en jeu des objets de chefs (dent de cachalot ou tabua) qui s'échangent "à la manière des chefs ». Et, en continuité, les trajectoires de plusieurs dirigeants successifs après l'indépendance seront connectées à diverses interprétations, également plurielles, de leur mana. Ceci permettra de révéler la nouvelle configuration de la classe sociopolitique fidjienne dans toute sa complexité.

\section{« La chefferie ", une reconstruction occidentale}

J'ai montré ailleurs (Cretton, 2005, 2007, 2009) que la chefferie est une construction anthropologique qui a d'abord été considérée, dans une perspective néo-évolutionniste, comme un stade intermédiaire entre la tribu et l'État (Sahlins, 1961; Service, 1962). Essentiellement interprétée en opposition à l'État de droit, elle a été opposée à la démocratie occidentale, elle-même réduite à un système de vote, à la citoyenneté ou aux droits civiques. En général, du côté des administrations coloniales et postcoloniales successives, comme du côté d'une certaine littérature savante, les chefs fidjiens ont été perçus comme des entités figées et homogènes, des obstacles au développement économique et politique de l'archipel. Cette perception reflète une dichotomie particulièrement étayée en sciences sociales et hors les murs de l'académie, celle qui opposerait les sociétés traditionnelles aux sociétés modernes, les sociétés d'ailleurs à celles d'ici, hier à aujourd'hui.

\section{De l'ethnocentrisme à l'écocentrisme}

Du côté universitaire, Lawson (1991, 1996) a contribué à cristalliser cette opposition en avançant que la démocratie est "ce qui manque " à la politique fidjienne perçue comme l'apanage d'une élite de chefs voulant maintenir ses privilèges. Cette idée que les chefs coutumiers fidjiens freinent la croissance économique et politique de l'archipel remonte à la période coloniale. On se souvient du rapport Spate, commandité par l'administration britannique en 1958, qui remettait sévèrement en question le rôle des chefs, en cristallisant la dichotomie "individu versus collectif» et en renforçant une opposition entre "le système de l'argent" (individualisme) et celui de "la coutume" (collectivisme). Une autre expertise plus récente a conclu aux mêmes résultats, malgré 44 ans d'écart (Cretton, 2007 : 274-280). En 2002, le rapport de la société d'Audit Pricewaterhouse Coopers, mandatée par le gouvernement fidjien pour réviser l'administration fidjienne, a similairement incriminé « l'incompétence » des chefs coutumiers en matière économique et politique, leur " manque d'intérêt pour le peuple", leur autorité qui «ne peut plus aller de soi ", ou encore "les conflits entre les droits individuels et le système collectif de la vie au village». À nouveau, "tradition" et "culture" sont opposées au "progrès ", à « l'économie », au « développement ».

Avec récurrence, le système des chefs est désigné comme l'antithèse d'un système "économique " global et " démocratique " dans lequel pourtant il s'insère. Suivant le même esprit, au cours de la dernière décennie, une certaine littérature savante anglophone a diffusé l'idée que certains États sont plus faibles que d'autres, en raison de leur instabilité politique. Plus récemment encore, Robertson (2012) a mis en évidence "l'échec" des leaders fidjiens à démocratiser leur communauté depuis l'indépendance. Ce point de vue avait déjà été critiqué notamment par Douglas (2003: 8) qui a montré que " la plupart des ethnologues dépeignent l'identité nationale en Mélanésie comme récente, faible, contestée ou absente, surtout dans les régions reculées". En opposition, les États "forts" sont définis par l'idée d'une démocratie florissante et d'une 
croissance économique stable sur le plan national et mondial. Aujourd'hui, dans le contexte d'une économie décrétée globale, une graduation en terme de performance persévère à hiérarchiser les sociétés du globe - non plus de la sauvagerie à la civilisation, mais de la faiblesse à la force, selon des critères à la fois occidentaux, économiques et politiques.

Toutefois, plutôt que nous dire quelque chose de spécifique à propos du système de chefferie fidjien, ce type d'analyse exacerbe le biais ethno et/ ou éco centriste de son approche. Par exemple, en posant des questions sur les incompatibilités entre le développement économique de l'archipel et l'Administration fidjienne, les deux Audits réalisés par Spate et PricewaterhouseCoopers postulent un lien de causalité entre l'un et l'autre, mais ce n'est jamais la croissance économique qui est perçue comme une entrave à l'Administration fidjienne. Ce n'est jamais le système de "développement économique " dominant qui est remis en cause, mais bien l'Administration fidjienne et le système de chefs sur lesquels elle se fonde.

Sans prendre en considération la spécificité du "profit» dans son acception fidjienne (redistribution plutôt qu'accumulation), ni même reconnaître la complexité de la société insulaire, les rapports d'expertise réduisent celle-ci à ses "faiblesses ", à ses "manques ", à ses "rigidités ", en regard du système économique dominant. Il est implicitement postulé que la société fidjienne "doit " s’insérer dans ce système plus vaste, sans remettre en cause celui-ci. En clair, une autre vision du développement économique et de l'économie en général - qui ne serait pas calquée sur les modèles du libéralisme - n'est jamais prise en considération dans les discours dominants (ce qui n'est bien sûr pas une spécificité fidjienne). Par contre, cette définition par l'" absence » révèle les enjeux idéologiques sousjacents aux réflexions scientifiques, aux expertises ou aux opinions produites sur ce sujet. À vrai dire, ce type d'analyse n'est pas nouveau, puisqu'il avait déjà été critiqué par Clastres (1974), lorsqu’il relevait le biais ethnocentrique des théories sur les sociétés « sans État ».

Ce modèle de réflexion par " le manque " a pris et prend toujours pour référence l'État, la structure, les valeurs ou encore les croyances des anciennes sociétés coloniales pour mesurer la progression politique, sociale, ou économique des autres sociétés. Dans le cas fidjien, ce point de vue est celui qui a remodelé l'archipel dès 1874 , lorsque les administrateurs britanniques uniformisèrent les structures fidjiennes existantes dans l'objectif de développer la colonie, important d'Inde une maind'œuvre considérée comme propice au travail dans les plantations decanneà sucre, confinant les Fidjiens dans leurs villages et renforçant les privilèges de leurs chefs en instaurant un grand conseil des chefs.

\section{La restructuration coloniale: simplifier la diversité}

Il a été démontré que la restructuration coloniale de la société fidjienne a contribué à son homogénéisation (Lal, 1992). Par exemple, les diverses chefferies existantes avant l'arrivée des Européens ont été regroupées en trois grandes confédérations ou matanitu (Kubuna, Tovata, Burebasaga), considérées comme les unités les plus importantes sur le plan coutumier ${ }^{1}$. Le matanitu a représenté " la forme la plus élevée d'organisation politique fidjienne" (Nayacakalou, 1975: 23)². Celle-ci résulte d'un long passé de querelles, d'alliances et d'obligations entre chefs, missionnaires et colons (Routledge, 1985; Tuwere, 2002 : 2430). Aujourd'hui, ces trois confédérations divisent l'archipel en trois grandes chefferies, si l'on peut dire, et dans l'ensemble, les sources convergent pour admettre l'uniformisation d'une structure sociale autrefois flexible et diversifiée (France, 1969; Nayacakalou, 1975, 1978 ; Ravuvu, 1983, 1991 ; Lawson, 1991 ; Robertson et Sutherland, 2001).

Nayacakalou (1975: 38-40) cite plusieurs exemples de chefs étrangers adoptés par un groupe, ou encore de chefs usurpateurs ou de chefs chassés par les leurs. Le principe de l'usurpation de pouvoir est fréquent dans les traditions des états tributaires et Fidji n'est pas une exception (Rutz, 1995 : 76). En ce sens, l'administration coloniale a fortement restreint la liberté de choisir un leader (Nayacakalou, 1975 : 40). Elle a simultanément contraint une hiérarchie autrefois mobile à se conformer à des règles de descendance lignagère patrilinéaire. Ces règles furent énoncées à des fins de répartition foncière pour mieux pouvoir administrer la colonie. Le critère de descendance patrilinéaire a été sélectionné par l'administration Gordon pour déterminer à la fois des unités parentales de propriété foncière et une hiérarchie de rang (chef/non-chef), en évacuant la diversité des situations existant à l'époque, spécialement à l'intérieur et à l'ouest de Viti Levu. Toutefois, une certaine variété semble perdurer, selon diverses procédures d'affiliation individuelles et collectives sous-jacentes à tel ou tel groupe.

Divers groupes de parents, dans de nombreux villages, ne connaissent pas leur ascendance originelle précise. Il n'est pas rare non plus qu'un groupe de parents qui ignore son ancêtre commun soit rattaché à un groupe quelconque, par adoption ou simple affiliation. Nayacakalou (1975:39) cite l'exemple du district de Tokatoka qui réunit six villages dont deux villages de chefs ont été adoptés

1. Dans son sens " moderne " et courant, matanitu signifie " un État ", " un gouvernement ".

2. Nayacakalou relate l'existence supposée de huit ou neuf matanitu à Fidji avant l'intrusion politique des Européens. Lauteur mentionne qu'il est difficile de définir ces organisations avec précision, car elles étaient sujettes à des alliances politiques particulièrement fluctuantes et changeantes dans les turbulences du XIX ${ }^{e}$ siècle. 


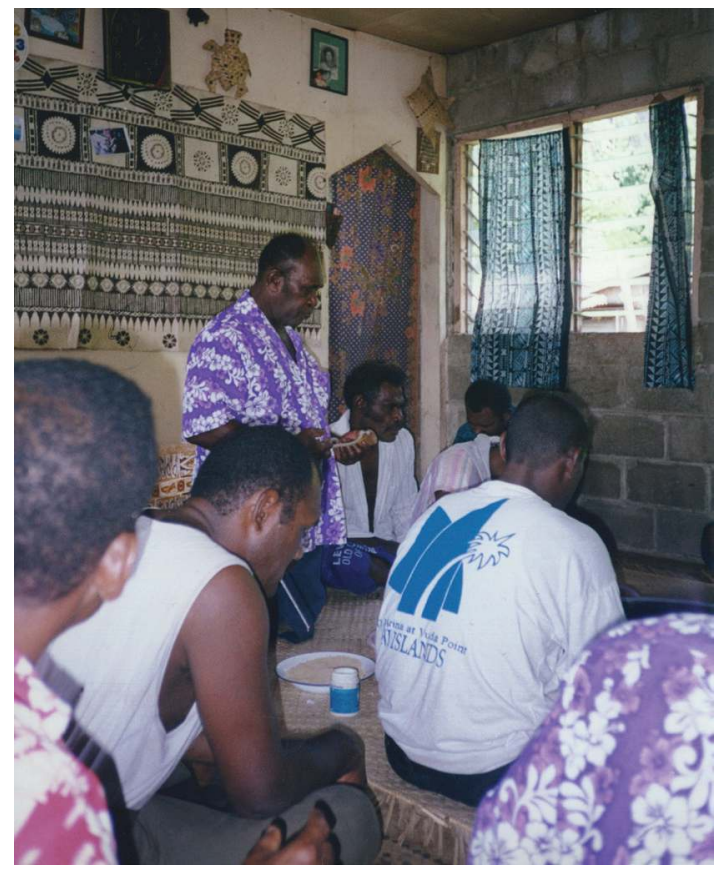

Рнотоs 1-2. - Échange de tabua lors de la restitution d'un couple dans son village, Moturiki (clichés de l'auteur, 2000)

par le groupe plus large. Des situations similaires sont rapportées par Geddes sur l'île de Vatulele (cité par Nayacakalou, 1975 : 39).

\section{Une structure mille-feuilles}

Chefferie et État sont en quelque sorte imbriqués, depuis l'indépendance en 1970 au moins. À Fidji, comme dans d'autres sociétés postcoloniales, différents types de pouvoir, anciens et nouveaux, sont à prendre en considération. Ceux-ci tantôt se superposent ou s'enchevêtrent, tantôt se confrontent ou se côtoient. Le rôleque peuvent jouer les hiérarchies fidjiennes dans les "structures constitutionnelles modernes " a été particulièrement mis en évidence par l'historien samoan Tuimaleali'ifano (2000: 253) :

« les liens du sang, les mariages politiques ou dynastiques, la religion, les capacités personnelles et les richesses relatives sous-tendent la suprématie politique et économique."

La façon dont les relations de parenté dites traditionnelles sont impliquées dans les affiliations politiques contemporaines complexifie les relations actuelles et leur compréhension pour l'analyste, car différents niveaux de références (anciens et nouveaux) peuvent s'y superposer ou s'y confondre. Les anciennes querelles pour la suprématie à Fidji peuvent fournir des informations sur les chicanes politiques actuelles entre divers partis. J'ai montré ailleurs (Cretton, 2007: 167-216) comment d'anciennes rivalités entre les chefferies de Bau, Lau

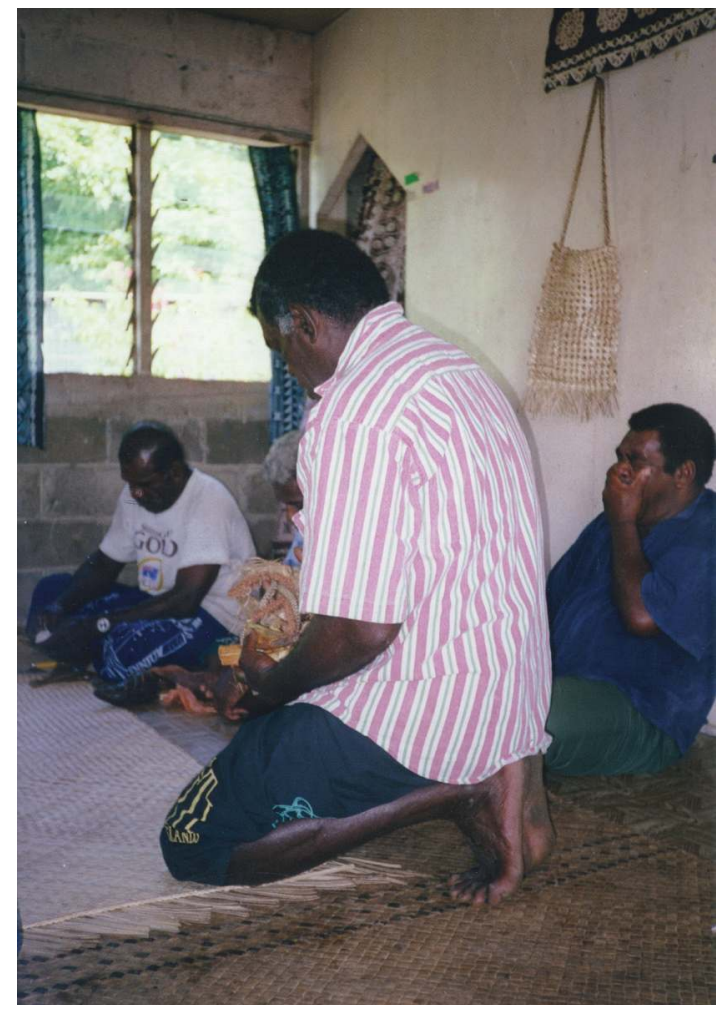

et Cakaudrove se sont rejouées lors de la destitution de feu Sir Ratu Kamisese Mara, Président de Fidji en mai 2000. A contrario du discours dominant, des relations de rivalité - veiqati - entre Lau et Bau pour la course au titre de Tui Kaba témoignent de la complexité de la situation politique fidjienne qu'il serait réducteur de résumer à la survivance d'une vieille querelle coloniale entre l'Est et l'Ouest. En effet, les rivalités entre les deux lignages de la famille Cakobau - les Mataiwelagi et les Naisogolaca qui se sont réactualisées suite au coup d'État en 2000 incarnent plutôt le dynamisme du paysage politique à Fidji, ses variations, ses mouvements, sa profondeur et sa complexité.

Tenter de comprendre le pouvoir politique à Fidji sans prendre en considération la multiplicité des niveaux de référence qui s'incarnent dans les relations (politiques, de parenté, sociales, genrées, ethniques), ainsi que les diverses périodes historiques qui structurent cette société (précoloniale, coloniale, postcoloniale) serait vain, ou pour le moins réducteur. Sur un plan générique, le chef compris comme figure du pouvoir - se positionne aujourd'hui à l'intersection des cultures politiques locales, nationales et globales (White et Lindstrom, $1997: 3)$.

\section{Chief, une catégorie plurielle et située}

Ce n'est qu'aux $\mathrm{XVIII}^{\mathrm{e}}$ et $\mathrm{XIX}^{\mathrm{e}}$ siècles - période majeure de l'exploration européenne et de la colonisation dans le Pacifique -, que "chef" (chief) est devenu le terme courant pour nommer 
les dirigeants " des peuples sauvages et barbares " (White et Lindstrom, 1997 : 7) du Pacifique.

Aujourd'hui, le "chef" peut se comprendre comme un leader politique qui a construit son autorité et son influence à partir d'un discours issu de la tradition locale (ibid.: 10). Suffisamment souple, cette catégorisation permet d'inclure ce que Sahlins (1968) a appelé bigman, cette figure de pouvoir comparée au "bourgeois" occidental et devenue emblème du système politique propre à la Mélanésie. La distinction faite entre bigman mélanésien (pouvoir acquis) et chief polynésien (pouvoir hérité) présente une typologie dualiste qui simplifie, en quelque sorte, les variations multiples de la réalité du leadership dans le Pacifique Sud (White et Lindstrom, 1997: 9). À Fidji notamment, acquisition personnelle et héritage sont étroitement imbriqués. Ceci pourrait s'expliquer par le positionnement de l'archipel, au croisement de la Mélanésie et de la Polynésie. Mais des imbrications similaires sont observées entre autres en Papouasie Nouvelle-Guinée, Vanuatu et Samoa.

Chez les Sulka de Nouvelle Bretagne orientale, par exemple, on ne devient pas taven (chef de village, statut le plus élevé qui a cependant perdu sa validité aujourd'hui) parce qu'on a réalisé de grandes choses, mais on réalise de grandes choses parce qu'on est taven (Jeudy-Ballini, 2004 : 116119). Ici, le statut de taven est d'abord hérité avant d'être démontré, c'est-à-dire collectivement validé (ibid.). Au Vanuatu en Mélanésie, les $\mathrm{Ni}$ Vanuatu s'attribuent le terme "chef " (ou chief en anglais et jif en bislama) dans divers contextes politiques ou économiques (Wittersheim, 2002). Mais le champ sémantique de la notion est on ne peut plus flou et chief désigne peutêtre tout simplement " un leader, quel qu'il soit " (ibid. : 135). En Polynésie, à Samoa, le matai est librement choisi comme représentant du groupe familial (Tcherkézoff, 2000 : 115).

En fait, de nombreux chefs polynésiens aujourd'hui manifestent des capacités personnelles qui rentreraient dans la catégorie de bigman, comme c'est le cas à Samoa. En parallèle, un certain nombre de leaders des sociétés mélanésiennes affiche des caractéristiques qui les rattacheraient à la catégorie de chief, comme c'est le cas chez les Sulka cités plus haut. À vrai dire, la catégorie de bigman n'est plus guère utilisée que par quelques anthropologues (White et Lindstrom, 1997; Wittersheim, 2002) pour signifier tantôt un type de pouvoir acquis, tantôt un modèle de pouvoir propre à la Mélanésie. Et la plupart des dirigeants $\mathrm{du}$ Pacifique postcolonial réclament pour euxmêmes le titre de chief, comme c'est le cas au Vanuatu ou à Fidji.

À Fidji, en général, chief réfère à l'héritage du titre de chef, mais celui-ci doit susciter l'accord du groupe concerné et peut se refuser par l'individu prétendant au titre. Le langage courant oppose le chief au commoner, compris comme n’appartenant pas à une lignée de chefs et ne possédant pas de titre. Toutefois, la signification exacte du terme " chef " differe d'un lieu et d'une communauté à l'autre. En général, un chef est un turaga. C'est-à-dire quelqu'un qui possède le titre de chef, soit parce qu'il appartient à une famille de chef, soit parce qu'il a été installé rituellement en tant que chef dirigeant de son groupe. Hérités de façon patrilinéaire depuis la colonisation (1874-1970), les titres varient selon les régions de l'archipel : le plus répandu est Ratu pour les hommes. Les femmes de lignée de chef possèdent le titre de Adi. Dans les provinces de Rewa et Naitasiri, le titre équivalent est $R o$ pour les hommes comme pour les femmes. Il reste difficile d'établir les terminologies emic utilisées avant l'arrivée des Européens. Sans doute que le terme originel était Tui, de Tù qui signifie littéralement " qui se tient dans la place " et que l'on retrouve dans $R a$-tì, où $r a$ désigne une marque de respect envers celui " qui se tient dans la place ». Tui est aujourd'hui le titre le plus élevé associé à celui de king, ou roi. Le titre de Tui est octroyé aux chefs (hommes et femmes) de lignages importants et aux chefs des trois matanitu ou grandes confédérations de Fidji : Burebasaga, Kubuna, Tovata. Chacune d'entre elles possède un titre suprême : Tui Dreketi pour Burebasaga $^{3}$, Tui Kaba pour Kubuna ${ }^{4}$ et Tui Cakau pour Tovata 5 .

À travers la littérature, le plus célèbre des chefs fidjiens est peut-être Ratu Seru Cakobau, surnommé Tui Viti, le grand roi de Fidji. Il vécut de 1815 à 1883 et céda Fidji à la Grande-Bretagne en 1874, en collaboration avec onze autres chefs de Fidji. Toutefois, à la différence de Kamehameha V à Hawaï et malgré son surnom, sa suprématie n'a jamais suscité l'unanimité du peuple fidjien et Tui Viti n'a jamais fait l'objet d'une installation rituelle'. Aucun individu n'ayant été installé rituellement en tant que "chef de Fidji ", d'anciennes rivalités perdurent entre les trois grandes confédérations de l'archipel, à propos de la suprématie du titre. Voyons de plus près comment celles-ci se sont

3. Lépouse de feu Ratu Mara, Ro Lala Mara fut titulaire du titre de 1974 à 2004. L'actuelle titulaire du titre est Ro Teimumu Kepa.

4. Titre vacant et disputé.

5. Ratu Naiqama Lalabalavu détient ce titre. Il reste cependant d'autres porteurs du titre de Tui, comme Tui Soso, Tui Tubou, qui peuvent par ailleurs être des " commoners ", même s'ils ne l'ont probablement pas toujours été.

6. Par contre, en tant que chef suprême du matanitu de Kubuna, Ratu Seru Cakobau était détenteur du titre de Tui Kaba Na Vunivalu. 
exprimées et recomposées au niveau politique, suite au coup d'État de 2000.

\section{"Chefferie » et hétérogénéité. Ou des divisions entre les chefs de l'Est, ceux de l'Ouest, du Nord et le grand conseil des chefs}

Suite à la prise en otages de plus de trente parlementaires fidjiens et indo-fidjiens en train de siéger dans une salle du Parlement à Suva, le 19 mai 2000, la plupart des chefs de villages, de districts et de provinces se sont prononcés dans la sphère publique pour faire part de leur position : pour ou contre le renversement du gouvernement par George Speight et son groupe armé. Cet événement a dès lors révélé des divisions anciennes et nouvelles entre les différents matanitu de Fidji. Des revendications pour une quatrième confédération (matanitu) dans l'ouest de l'archipel ont ressurgi, ce qui avait été aussi le cas en 1987. Ces prises de position publiques - sous forme de communiqués de presse, de prises de parole dans les médias ou de manifestations - ont réactivé une vieille division coloniale entre les chefs de l'Est Fidji et ceux de l'Ouest. Toutefois, ces dissensions ne peuvent pas s'expliquer comme étant des survivances d'un passé colonial voire précolonial. Elles illustrent plutôt l'expression d'un mécontentement, certes historiquement construit, mais inséparable aujourd'hui de la situation économique. Regardons cela de plus près.

\section{Rivalités historiques, symboliques, économiques}

Les 7 et 8 juin 2000, de nombreux chefs venus des provinces de Namosi, Serua, Nadroga/Navosa, $\mathrm{Ba}$ et Ra dans l'ouest de Viti Levu - appartenant aux confédérations de Kubuna et Burebasaga - se sont réunis à Nadi pour discuter la constitution d'une quatrième confédération dont le nom serait Yasasa Vaka Ra. Préalablement, entre le 21 mai et la fin du mois de juin 2000, diverses délégations de chefs de l'Ouest se sont exprimées dans les médias pour évoquer la "suprématie " des chefs de l'Est Fidji en matière de politique nationale.

«D'abord le gouvernement dirigé par feu Timoci Bavadra et maintenant c'est le gouvernement de Chaudhry qui a été renversé, arme au poing. Bavadra était de Viseisei et Chaudhry est de Ba. Il semble que ces bandes qui ont renversé le gouvernement élu démocratiquement en 1987 et cette année n'aiment pas les dirigeants de l'Ouest. Alors la seule chose à faire est de former un gouvernement à l'Ouest. " (Ratu Jone Ravetale, chef du village de Navoci, Fiji Sun, 6 juin 2000)
Feu Dr. Timoci Bavadra, Premier ministre en 1987, était originaire du village de Viseisei dans la province de $\mathrm{Ba}$, à l'Ouest. Il a été destitué par le Colonel Sitiveni Rabuka, originaire de Cakaudrove (sur l'île de Vanua Levu, dans la division Nord de Fidji). En 2000, le Premier ministre Mahendra Chaudhry, aussi originaire de $\mathrm{Ba}$, a été destitué par George Speight, originaire de Tailevu à l'est de Viti Levu. Mais les revendications pour un gouvernement séparé, par les chefs de l'Ouest, dépassent les origines régionales des leaders. En 2000, elles se sont principalement fondées sur l'importante contribution de l'ouest du pays à l'économie nationale. En réalité, les chefs réclamaient une représentation sur le plan de la politique nationale qui soit proportionnelle à leur apport à l'économie du pays :

«Nous avons l'industrie du sucre, les mines d'or, l'aéroport international et les attractions touristiques majeures. "(Ratu Sairusi, chef de Ba, Fiji Times, 4 juin 2000)

En 1987 déjà, des requêtes similaires à celles de 2000 s'étaient exprimées dans l'Ouest, plaçant la question économique au cœur des revendications pour une quatrième confédération, (Thomas, 1990 : 137). Â ce moment-là, les gens " ordinaires " (non-chefs) espéraient profondément qu’un gouvernement autonome leur permette de se faire entendre à Suva, la capitale. Cela aurait pu conduire, pensaient-ils, à construire davantage de routes et à fournir d'autres services nécessaires dans la région. En 2000 par contre, les revendications pour une quatrième confédération ont plutôt porté sur la possibilité d'une autonomie économique de l'Ouest par rapport au reste du pays :

"Ce qui nous concerne en priorité, c'est que l'Ouest a toujours été affecté. Nous souffrons le plus parce que la plupart de nos personnes sont employées dans le tourisme et l'industrie du sucre - deux industries qui soutiennent l'économie nationale. Et certains sentent que nous n'avons pas à subir les difficultés créées par les autres, dès lors que nous avons les ressources pour nous assumer nous-mêmes.» (Ratu Osea Gavidi, chef de Nadroga, Fiji Times, 8 juin 2000)

La division entre l'est et l'ouest de Fidji s'inscrit en continuité avec un passé précolonial socialement diversifié (Thomas, 1990 : 132), constitué d'alliances et de relations changeantes. Il est communément considéré que dans les petites îles qui constituent le centre et l'est de Fidji, des confédérations hiérarchisées se sont développées et que les plus grands de ces chefs ont plus tard été impliqués dans l'administration coloniale. À l'intérieur et à l'ouest de Viti Levu, par contre, le pouvoir traditionnel aurait été plus localisép 
De substantielles rébellions ont toutefois eu lieu à l'intérieur de l'île, nécessitant une répression militaire organisée pendant la colonie (Ravuvu, 1974: 4). En 1876, également, des gens de l'intérieur (kai Colo, hill tribes) de l'île principale se sont soulevés pour revendiquer leur autonomie politique et religieuse, en s'opposant à la fois à l'administration coloniale et à la tentative de conversion au christianisme (Thomas, 1990 : 134). Cette insurrection s'inscrivait également dans un passé de guerres internes. Le conflit a opposé les gens de la côte ouest et du bas de la vallée de Sigatoka et ceux de l'intérieur, à l'ouest du pays.

Cette division entre les gens "de l'intérieur" et ceux "des côtes" persiste dans les affiliations et les alliances contemporaines : les gens du sommet de la vallée sont étroitement reliés à ceux de l'intérieur mais pas à ceux de la côte (Thomas, 1990). La situation s'est complexifiée avec l'administration coloniale qui regardait les gens des collines (kai Colo) comme des semeurs de troubles. Celle-ci
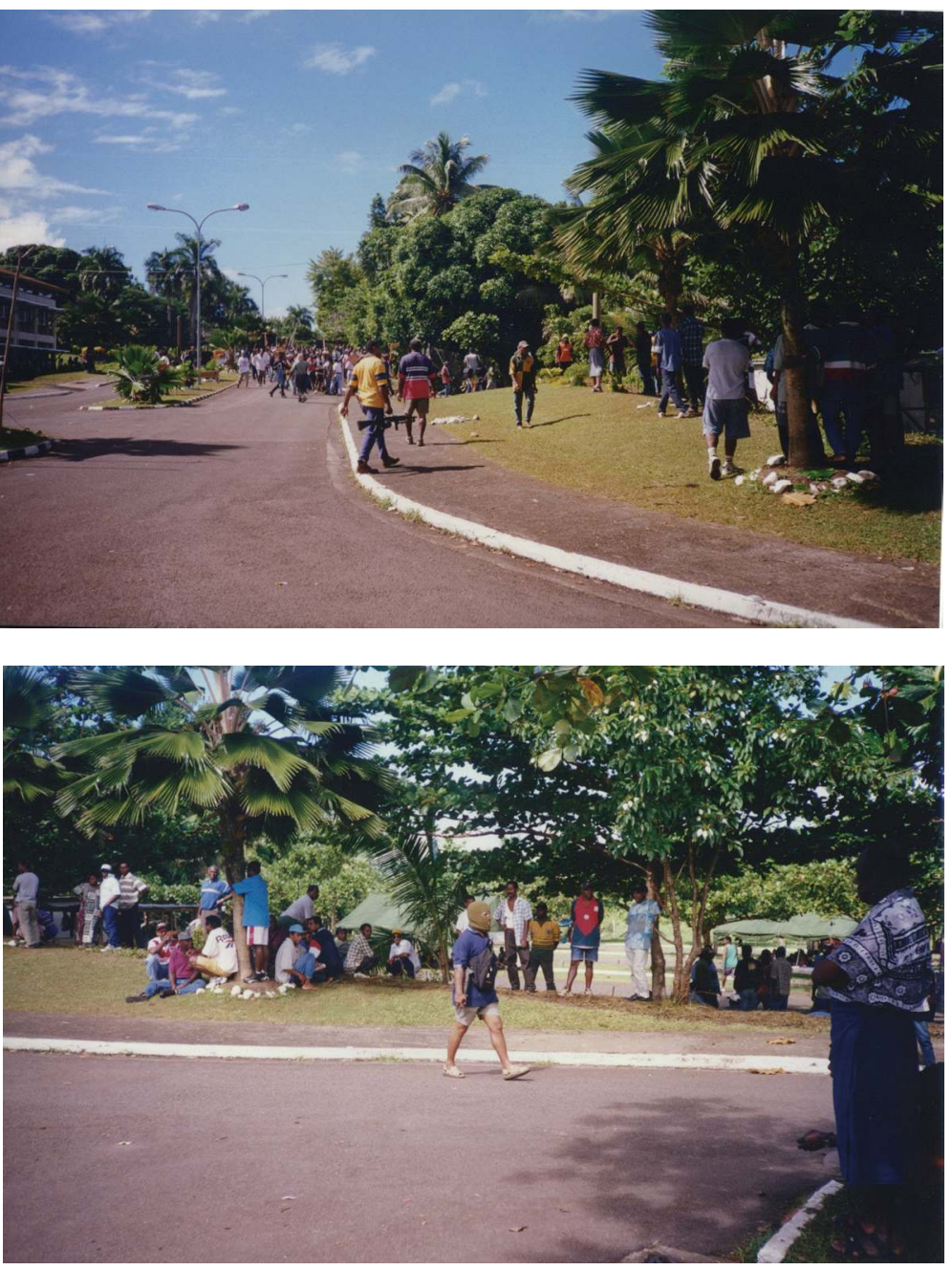
tenta alors de diviser le groupe "de l'intérieur" (bighlanders) relativement uni en trois provinces : Colo East, Colo West et Colo North. Lors d'une réorganisation $\mathrm{du}$ système administratif concernant les Fidjiens autochtones en 1948, ces provinces furent assimilées à des provinces de la côte (Thomas, 1990 : 134). Ceci activa le mécontentement des gens de l'intérieur qui, désavantagés économiquement, se retrouvèrent marginalisés au sein de leur nouvelle province.

À la suite de 1987, il avait été proposé que la présidence de la République circule entre les chefs suprêmes de Tovata, Burebasaga et Kubuna, sur une base de cinq ans. Mais la partie occidentale de l'archipel se répartissant entre les confédérations de Burebasaga et Kubuna, les dirigeants de l'ouest se sont sentis fortement marginalisés par cette proposition (Thomas, 1990 : 137). En 2000, ces dissensions internes, exacerbées, se sont exprimées par des manifestations de ralliement ou de résistance à la " cause indigène » défendue par George Speight. Rendons-nous à l'intérieur du Parlement fidjien assiégé, à Suva, pour observer ceci de plus près.

\section{Médiations, transactions symboliques et gestion du conflit}

Quelques jours après la prise d'otages en mai 2000, George Speight et ses hommes ouvrirent les portes du complexe parlementaire à leurs supporters. Rapidement, l'événement se mua en une sorte d'attraction, éveillant la curiosité et le soutien d'un public de plus en plus nombreux et suscitant l'intérêt des médias nationaux et internationaux.

Des centaines d'hommes et de femmes, jeunes et moins jeunes, principalement issus des villages avoisinants, se rendirent dans l'enceinte du Parlement pour soutenir le coup d'État, en apportant diverses offrandes de nourriture et du kava. Des délégations et des médiateurs de toutes sortes se succédèrent pour venir négocier ou pour faire valoir leur position auprès des "rebelles" : la délégation du grand conseil des chefs ; la délégation des chefs de l'Ouest ; Ratu Tu'akitau Cokanauto, chef de Bau et leader du Fiji Association Party, venu boire le kava avec les auteurs du coup d'État, le 22 mai; les membres de la Counter Revolutionary Warfare Unit (CRWU) - une unité de 


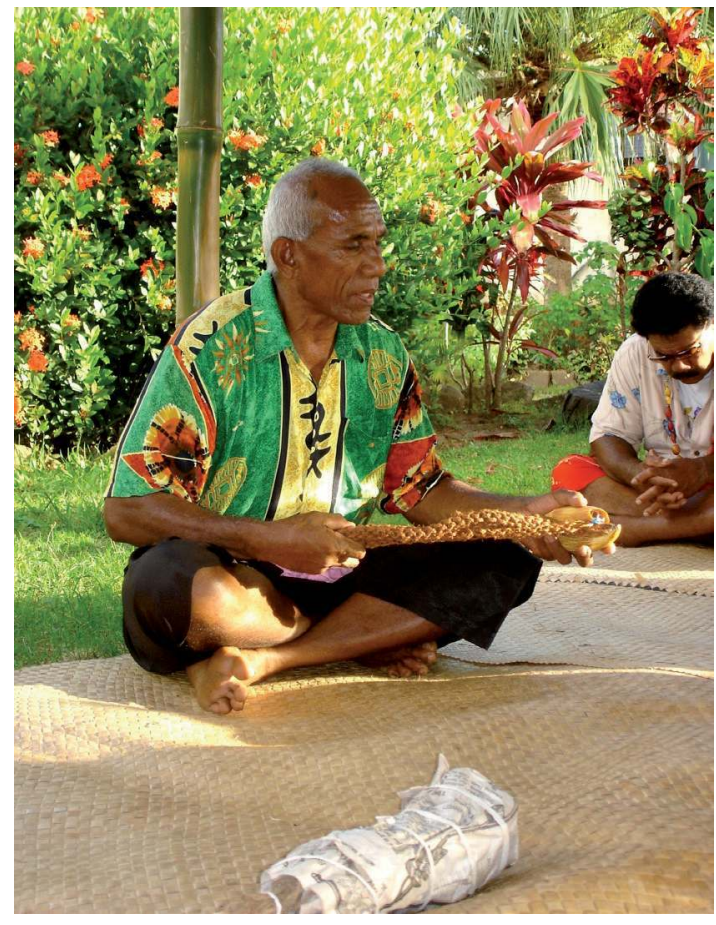

Рното 5. - Présentation d'une dent de cachalot à un chef (Lakeba, Tubou, octobre 2008, cliché de Simonne Pauwels)

milice spéciale au sein de l'Armée - qui se sont ralliés à Speight dès le début de la prise d'otages ; l'envoyé spécial des Nations unies, Serge Veira de Mello, et le secrétaire du Commonwealth, Don Mc Kinnon, venus ensemble tenter de négocier avec les «terroristes » et visiter les otages, le 24 mai ; les journalistes d'outre-mer nourris et logés par les rebelles dans un espace spécialement apprêté pour eux au sein du complexe parlementaire; les activistes Māoris s'étant expressément déplacés de Nouvelle-Zélande en signe de soutien à la cause indigène; la délégation de supporters du parti Soqosoqo ni Vakavulewa ni Taukei (svT, un parti défenseur des droits et intérêts des Fidjiens autochtones), menée par le maire de Suva, Mataiasi Ragigia, effectuant une visite dite " protocolaire ", le 4 juin ; la délégation de la très influente Église méthodiste comprenant le Révérend Anil Reuben, chef de file des Méthodistes indiens, et venant proposer une « médiation spirituelle ».

\section{Le grand conseil des chefs, une médiation}

C'est dans ce contexte que le grand conseil des chefs, dirigé par Sitiveni Rabuka, s'est profilé tel le médiateur principal des négociations. Au plan législatif, le grand conseil des chefs avait (avant son abolition) des devoirs de protection envers les Fidjiens. Mais cette responsabilité lui était communément attribuée au plan moral également. Dans la "philosophie fidjienne» (Durutalo, 2003 : 170), le rôle du chef est avant tout celui d'un "protecteur " et d'un "gardien de la paix " pour tous ceux qui vivent sous son autorité traditionnelle. Ceci implique que lors de conflits, le rôle du chef en tant que " pacificateur " et « arbitre neutre » devient crucial pour la stabilité de la vie dans la communauté. Il n'est donc pas arbitraire qu'après le coup d'État, le grand conseil des chefs ait été aussitôt investi du pouvoir (ou du devoir), par la population comme par ses élus, de réconcilier les différends liés à la crise politique.

De surcroît, les négociations qui ont mené à la libération des otages se sont déroulées suivant certaines caractéristiques qui n'ont rien à envier aux pratiques de participation citoyenne, telles qu'enseignées en Suisse notamment : une volonté rapidement activée de négocier entre groupes opposés, des pratiques mutuelles de respect entre factions ennemies, des échanges, de longues discussions en face à face souvent accompagnées de transactions symboliques. Parmi celles-ci, des échanges de tabua (dent de cachalot) ont joué un rôle spécifique durant le conflit de 2000.

\section{Les tabua: des objets de chefs, anciens, mythiques, et efficaces}

Les tabua incarnent le dynamisme et l'efficacité symbolique des pratiques dites coutumières au cœur du système de chefferie fidjien : le pouvoir de faire, refaire ou défaire les liens sociaux, celui de constituer des alliances ou d'ouvrir des discordes, celui de sceller la paix ou de déclencher le conflit. Considérée comme l'objet traditionnel suprême offert à la personne la plus importante, la dent de cachalot relève du don et du fait social total (Mauss, 1983/1950). Au cœur d'un mythe fondateur (Sahlins, 1982; Thomas, 1991), les tabua bénéficient assurément d'un statut symbolique privilégié à Fidji dont Mauss avait probablement déjà vu l'essentiel :

«Leur présentation constitue une requête; les accepter, c'est s'engager. » (Mauss, 1983 : 191)

Les tabua sont généralement présentées pour effectuer une demande individuelle ou collective à un individu ou à un groupe. Elles nécessitent un ou plusieurs donneurs et plusieurs receveurs. Accepter une tabua signifie accepter le dessein de la requête, qu'il s'agisse d'une demande spécifique ou d'une manifestation de bienvenue. La refuser consiste à refuser la demande en question. Dit rapidement, les tabua sont échangées dans le cadre de naissances, mariages, funérailles et d'autres événements comme la construction d'une maison, d'une église, d'une école, lors de la visite d'un personnage important ou encore lors de situations exceptionnelles.

Avant d'illustrer l'efficacité actuelle des tabua, il faut retenir que leur valorisation sociale et 
symbolique est telle qu'une demande impliquant une présentation de dent de cachalot est rarement refusée (Sahlins, 1962: 199) ${ }^{8}$, même si toutefois cela peut se produire (Hooper, $1982: 127-128$; Sahlins, 1991: 48 ; Waterhouse, 1997 : 79). Les événements de l'année 2000 montrent aussi que les refus de tabua sont possibles (Fiji Sun, 20 juillet 2000 ; Fijis Daily Post, 7 août 2000), mais qu'ils sont le plus souvent déclencheurs ou accélérateurs de conflit, à des degrés divers. Car ce que les refus remettent en cause, ce n'est pas simplement une demande quelle qu'elle soit mais plutôt ceux que cette demande engage soit les relations qui s'établissent entre le groupe des solliciteurs et celui des "refuseurs". Dès lors, pour appréhender le sens investi dans la transaction par les personnes concernées, il faut prendre en considération un réseau compliqué d'interactions agissantes, comme l'histoire, les relations de parenté et les dynamiques entre ces relations (Sahlins, 1962 ; Tuimaleali'ifano, 2000).

Pour exemplifier ce qui précède, en 2000, la destitution du président de la République des îles Fidji, Sir Ratu Kamisese Mara, s'est faite sur présentation de tabua. Le 29 mai au soir, le commandant des forces militaires fidjiennes, Frank Bainimarama, accompagné d'un groupe d'officiers, a approché le chef de haut rang de l'Est Fidji pour lui demander d'abdiquer. Lui présentant une tabua, il a sollicité son pardon pour cet acte de destitution. Le président a accepté (cf. Cretton, 2005 ; voir également Lal, 2000a : 281).

Subséquemment, sur l'île principale, plusieurs postes de contrôle policiers ou militaires et différents sites stratégiques (dont le barrage de Monasavu) ont également été occupés sur présentation de tabua, et de kava. Des groupes de propriétaires terriens ont bloqué plusieurs accès routiers aux alentours de Suva pendant des jours, en s'asseyant en tailleur à même le sol, tout en buvant du kava. À la fin de la prise d'otages encore (13 juillet 2000), différentes cérémonies impliquant des échanges de tabua ont eu lieu entre diverses factions pour demander pardon pour les violences commises et les dommages engendrés pour le pays, ainsi que pour réconcilier les différents groupes en présence.

\section{Des objets de tempérance}

Pour l'esprit acquis aux idées d'interventions armées nécessaires en situation de conflit, de telles pratiques de négociation (impliquant $\mathrm{du}$ temps, des transactions symboliques, une quête de consensus) peuvent être mal comprises. Par exemple, dans le cadre des pourparlers entre chefs, militaires et rebelles qui ont précédé la libération des otages, les présentations faites par la délégation des chefs au groupe de Speight ont été interprétées par des journalistes d'outremer comme un ralliement des chefs à la cause de Speight. Pourtant dans le contexte fidjien, offrir du kava, des nattes, des tissus d'écorce ou autres objets de valeur symbolique, et accepter ce qui a été donné ne signifie pas, de fait, "manifester sa sympathie", son "soutien" ou sa "reconnaissance», comme on pourrait le comprendre en Europe, où l'on donne rarement quelque chose à un ennemi ou à une personne qu’on n'apprécie pas. Pour l'individu façonné par des normes de socialisation individualiste, cette façon de négocier ne va pas de soi. Pour préciser ceci, écoutons M. Sadole qui faisait partie de la délégation du grand conseil des chefs durant les négociations :

"Chaque jour, nous [délégation du grand conseil des chefs] allions là-bas [dans le Parlement pris en otage], chaque jour, pour parler à ces personnes en essayant de résoudre la crise. Nous apportions du yaqona et des cigarettes à ces personnes qui faisaient partie de nos familles, parce que c'est la façon de faire dans notre culture. Alors les gens et les journalistes ont dit après que nous soutenions le coup d'État, mais non, en aucune façon. C'est un malentendu à propos de notre culture. Vous savez, je n'aime pas les journalistes étrangers, ils ont interprété que nous avons soutenu le coup d'État, mais non. Voyez, dans la culture fidjienne, c'est la façon de faire, offrir du kava et des cigarettes et quelques chocolats, parce que nous avions des membres de notre famille là-bas, aussi bien des personnes impliquées dans le coup d'État que des otages. Chaque jour, nous avons apporté du kava, nous nous sommes assis et avons parlé. Je me rappelle que le premier jour où nous avons dû y aller, ils étaient proches de se battre, mais le Président qui était très intelligent a dit non, maintenant nous devons régler cela, nous préparons le grog et nous allons boire le grog et après la première tournée de bols, vous savez, les gens se sont calmés [en descendant ses bras pour signifier le fait qu'ils s'étaient calmés]. [Rires]. Alors nous avons bu du grog, tous les jours nous avons bu du yaqona. C'est la façon fidjienne, vous savez, parce que lorsque vous buvez, vous montrez votre respect... Et probablement que si la crise avait été réglée par la loi, cela aurait engendré davantage de blessés. Vous savez, nous Fidjiens, nous buvons du kava en montrant notre respect [et il frappe trois fois dans ses mains]. » (Entretien avec M. Sadole, secrétaire du GCC, Suva, 6 août 2002)

Pendant les presque deux mois qu'a duré la prise d'otages, les objets symboliques qui ont circulé entre les principales catégories d'acteurs en présence ont eu pour effet d'activer les négociations politiques et de tempérer la violence du conflit de longs jours durant. On peut dire que les échanges de dents de cachalot incarnent la relation que

8. Sa valeur économique est également élevée aujourd'hui et les tabua, qui sont faites d'ivoire précieux, peuvent se vendre et s'acheter dans les divers pawnshops (monts-de-piété) de la capitale pour un montant qui peut varier entre 100 et plus de 700 dollars fidjiens (entre 40 et 300 euros selon les cours), ce qui est une somme importante à Fidji. 
les négociateurs fidjiens entretiennent avec le conflit et la violence, une relation pour laquelle tous les participants adoptent une attitude de chefs, vakaturaga: pour négocier les événements, amis et ennemis s'asseyent autour du kava et se manifestent un respect mutuel, s'élevant ainsi tous au rang de chef.

\section{"À la manière d'un chef"}

Littéralement, vakaturaga signifie qu'une action et ses caractéristiques satisfont la présence d'un chef (même s'il n'y a pas de chef à ce moment-là), parce que l'action est menée " à la manière d'un chef ». Ce comportement idéal se manifeste par des attitudes de respect (veidokai), de déférence (vakarokoroko), d'attention et d'obéissance (vakarorogo), ou d'humilité (malua), non seulement envers les chefs mais à l'égard de tout un chacun (Ravuvu, 1983 : 103-106). On dit d'une personne qui présente de telles caractéristiques qu'elle est tamata $i$ tovo vakaturaga, c'est-à-dire que son comportement satisfait la présence d'un chef, parce qu'elle agit comme si tout un chacun était quelqu'un d'aussi important qu'un chef ou qu'un supérieur :

"Lorsque vous êtes vakaturaga, vous ne montrez pas votre statut. " (Epi Duravasi, conseiller à l'École Queens Victoria, entretien, 26 août 2002)

À Samoa également, la position du chef n'est pas communément perçue comme étant hiérarchique et dominante, mais comme égalitaire et démocratique (Tcherkézoff, 2000). Pour les Samoans, la notion de matai ou de détenteur du titre est égalitaire parce que le matai est librement choisi comme représentant du groupe familial. Ce choix démocratique est fait par consensus et non pas par système de vote. Certes, avec le consensus, les personnes influentes ont naturellement un poids inégal en comparaison des jeunes ou des nouveaux, et cela peut paraître non démocratique. Mais de nombreux Samoans considèrent que le système de vote est désavantageux, parce qu'il réduit l'expérience de chaque personne. Beaucoup pensent que le système de vote introduit une dichotomie entre le gagnant et le perdant, ce qui crée une division au sein du groupe. Par contre, lorsqu'une décision est prise par consensus, il n'y a pas clairement de perdant. Ceci explique pourquoi le consensus est toujours la seule procédure admise dans toutes les assemblées de famille à Samoa (Tcherkézoff, 2000 : 115-116).

\section{Des objets d'échange spécifiquement fidjiens}

Aussi surprenantes qu'elles peuvent paraître à l'observatrice occidentale, les pratiques observées incarnent sans doute la spécificité du conflit fidjien de 2000 : un conflit dont la violence a été

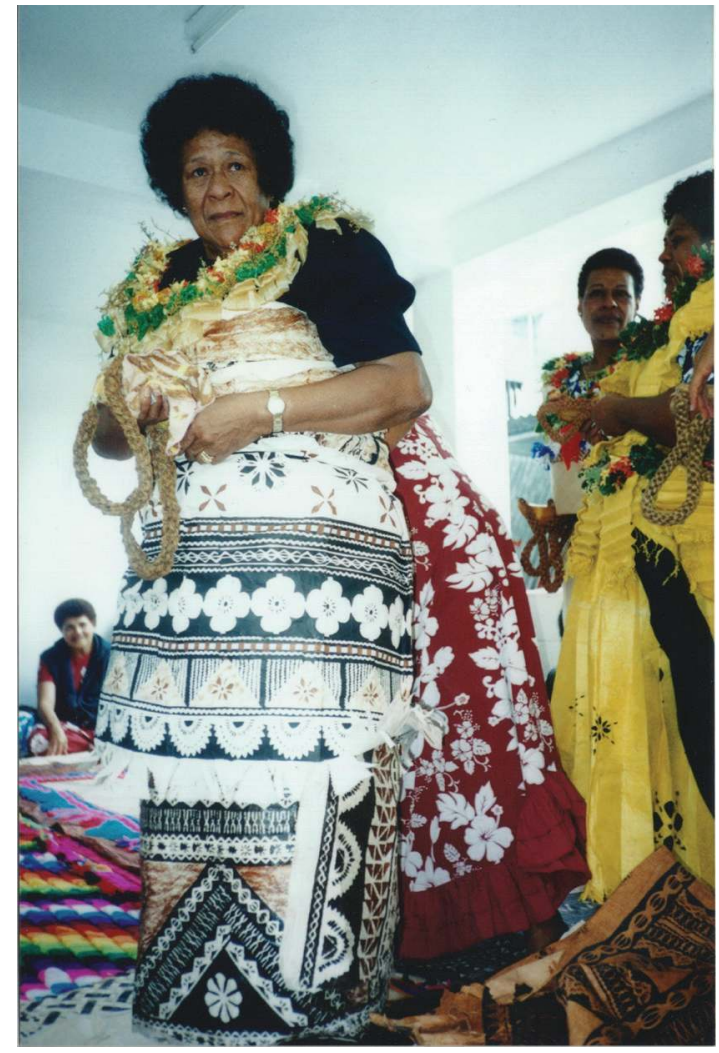

Рното 6. - Présentation de tabua par des femmes, lors d'une cérémonie de levée de deuil (cliché de l'auteur)

atténuée par de multiples échanges symboliques et pratiques dites "traditionnelles" ou "de respect " qui se sont déroulées entre les divers protagonistes. Le fait de manger et de boire la nourriture et la boisson "de la terre" (racines et yaqona) au pied du Parlement assiégé visait à manifester le soutien des "gens de la terre " (taukei) à la prise d'otages, tout en affirmant les valeurs du vanua (la terre et ses habitants).

Ailleurs dans le Pacifique Sud, ce ne sont pas des dents de cachalot mais d'autres biens qui sont mis en cause dans les cérémonies de conciliation: des dents de dauphins, des coquillages, des nourritures, des noix de bétel ou des nattes. Au-delà de l'objet particulier mis en jeu dans l'échange, Fidji se rattache aux sociétés du Pacifique Sud comprises comme des communautés qui connectent le social, le politique et l'économique sur fond de réciprocité. Toutefois, dans les îles avoisinantes, les pratiques de résolution du conflit peuvent contenir de fortes doses de compromis et de marchandage, en contraste avec les lois occidentales qui adjugent un vainqueur et un perdant (Dinnen, 2003 : 8). Aux Salomon, par exemple, dans le cadre d'un conflit accru entre 2000 et 2003, certaines pratiques coutumières de compensation ont pris des proportions exacerbées (Arkwright, 2003: 177-189). 
Traditionnellement, la compensation signifie le paiement cérémoniel de biens coutumiers en monnaie locale comme un moyen de redresser l'insulte faite au groupe. Mais dans la situation aux Salomon, il sagissait de réclamer le coût des destructions occasionnées par le conflit. Le père Arkwright (2003) raconte l'anecdote de 5 millions de dollars (en dollars des îles Salomon) demandés en compensation pour une injure dite à l'égard des gens de Malaita par les gens de Guanalcanal. Toutefois, à Fidji, la spécificité du $i$ soro ou cérémonie de réconciliation se distingue du rituel de compensation salomonais. Il s'agit plutôt d'une demande de réparation dans le sens de Hooper (1982 : 124), c'est-à-dire un sens très chrétien de demande de rachat.

Assurément, les transactions symboliques ont permis de pacifier le conflit en 2000 , de façon spécifique, tout en renforçant un sentiment d'appartenance identitaire aux sociétés du Pacifique Sud qui se sentent reliées entre elles par l'océan (Hau'ofa, 2008). Au cœur de ce système de don - porteur de revendications sociales et politiques face aux pressions internationales venues de l'onu ou du Commonwealth -, les relations de parenté et de pouvoir entre différentes catégories de chefs ou leaders ont révélé une autre dimension de la flexibilité inhérente à la chefferie fidjienne. Examinons cela de plus près.

\section{Figures de leader, conflits de classes, hétérogénéité du pouvoir}

Constitutives du politique à Fidji, les connexions de classes comme celles de parenté autorisent une mise en perspective de différents types de pouvoir, anciens et nouveaux, lesquels, en réalité, tantôt se superposent ou s'imbriquent, tantôt se confrontent ou se côtoient. Les figures de leaders successifs, légitimes et moins légitimes, exemplifient la diversité et le pluralisme inhérent à la catégorie de chef. Pour illustration, Ratu Mara et Rabuka incarnent deux figures de leader, l'une détentrice d'un titre élevé de chef et l'autre qualifiée de commoner.

Ratu Sir Kamisese Mara est originaire de la province de Lau, dans l'est de Fidji. Détenteur du titre de Tui Nayau, il est également soumis à l'autorité coutumière de Tui Cakau, pour le matanitu de Tovata. Chef de haut rang de l'Est Fidji, Mara a construit son $m a n a^{9}$ sur son propre titre et sur celui de sa femme, Roko Tui Dreketi, le titre le plus élevé de Burebasaga (Teiawa, 2001 : 31). Son éducation à Oxford lui a permis de faire carrière dans les affaires en s'associant avec une élite économique d'Européens et d'Indo-Fidjiens. En parallèle, sa carrière politique a évincé quelques chefs rivaux dans sa région d'origine, en générant simultanément un ressentiment dans les provinces situées à l'ouest (Teaiwa, 2001 : 31). Avec ses privilèges hérités de naissance, et son éducation universitaire valorisée symboliquement, Ratu Mara incarne une élite de chefs dont les pouvoirs ont été renforcés par l'administration coloniale, celle-ci ayant su ouvrer pour faciliter l'accès à l'éducation et à l'emploi (Leckie, 2002 : 128). Certains Fidjiens considèrent que les titres universitaires peuvent renforcer le mana d'un chef, spécialement lorsqu'ils s'acquièrent à l'étranger. Â Suva, plusieurs personnes m'ont parlé de Ratu Mara comme d'un chef possédant un double mana, c'est-à-dire une double autorité issue d'un double savoir. Il est perçu comme maîtrisant à la fois le langage de "la tradition" - en tant que Tui Nayau, chef de rang élevé du groupe des îles Lau - et le langage du monde politique ou économique occidental - en tant que président de la République, titulaire d'une licence d'économie obtenue à Oxford. Pour les gens des Lau, par contre, le mana provient uniquement du titre et de la relation avec les ancêtres. Ici, Ratu Mara est considéré comme efficace au plan de la nation grâce à son kaukauwa (force et pouvoir) et performant au plan de la chefferie, par son mana. Sur l'île de Lakeba, la construction d'un hôpital ou d'une école est considérée comme l'expression de son kaukauwa, pas de son mana. Par contre, le fait de ne pas être frappé par un cyclone ou d'avoir des jardins florissants sont des preuves du mana d'un chef ${ }^{10}$.

De même que le pluralisme et la complexité sont inhérents à la figure du chef à Fidji, polyphonie et nuances semblent constitutives des représentations locales et situées du mana.

Ainsi, tous les leaders politiques ne possèdent pas l'aura coutumière d'un chef de haut rang tel que Ratu Mara. Par exemple, l'ancien Premier ministre destitué en 1987, le Dr. Timoci Bavadra, est communément désigné par son titre universitaire de Docteur, et non pas en fonction de son origine coutumière dans le village de chef de haut rang de Viseisei, à l'ouest. Posséder un titre universitaire est généralement considéré comme un avoir efficace: celui de pouvoir se faire une place dans la société fidjienne. Ne pas être instruit du tout est souvent regardé, à l'inverse, comme quelque chose de péjoratif, au village comme en ville. Un anonyme instruit me confiait que le problème des chefs coutumiers,

9. Verbe signifiant « être efficace, réussir, être réalisé, "fonctionner" ». Keesing (1984 : 137) a montré que la traduction répandue de mana par une sorte de "médium invisible du pouvoir" est une erreur qui a donné lieu à de nombreuses mésinterprétations anthropologiques. Le mana est une condition et non pas une chose. Lorsque des choses sont mana, elles sont efficaces, vraies, performantes.

10. Je remercie Simonne Pauwels pour les précisions concernant Lau. 
" c'est qu'ils sont souvent ignorants ", c'est-à-dire qu'ils n'ont pas poursuivi leur instruction au-delà de la scolarisation secondaire, voire primaire. Selon cet interlocuteur, un certain nombre de chefs qui siègent au grand conseil des chefs n'ont pas bénéficié d'enseignement supérieur et se trouvent difficilement à même de décider, en ce qui concerne les questions de politique nationale. L'héritage du titre coutumier ne suffit plus à construire la légitimité du mana. $\mathrm{La}$ superposition des savoirs particuliers, transmis et acquis, inscrite dans un réseau de relations inter et intra-familiales, contribue à la (re)construction du mana, compris comme un pouvoir d'action politique efficace et performant, dans le sens de Keesing (1984).

Sitiveni Rabuka, quant à lui, est un bati (du clan des guerriers) ${ }^{11}$ de Cakaudrove qui a fait une carrière militaire dans l'armée fidjienne. Son mana s'est construit sur la ferveur religieuse qu'il a toujours manifestée publiquement et son soutien aux discours méthodistes. Ses deux coups d'État en 1987 et l'appui qu'il a constamment reçu du grand conseil des chefs ont participé à renforcer son autorité (Teaiwa, 2001 : 32). Diverses affaires privées médiatisées auraient de surcroît contribué à amplifier son charisme politique, en le rendant sympathique et accessible aux yeux de ses admirateurs. Les gens du peuple « peuvent s'identifier plus facilement à Rabuka qu'à l'aristocrate Ratu Mara " (Teaiwa, 2001: 32). À l'opposé de l'ancien Président Mara, Rabuka a développé une popularité de self-made man. Le gouvernement de Rabuka a permis l'accession d'une nouvelle "classe" de personnes - pas forcément titulaires de titre de chef - dans l'administration gouvernementale. En tant que commoner, le leader des coups d'État de 1987 a participé à la constitution d'une nouvelle mobilité sociale, qui trouve déjà certains antécédents sous la gouvernance de Ratu Sukuna - chef de Bau, et premier président à la tête de l'administration fidjienne en 1954.

\section{Du mana issu des classes moyennes}

L'expansion de la classe moyenne comprise comme l'avènement d'un groupe de fonctionnaires, travaillant dans les nouveaux départements gouvernementaux (Norton, 1977 : 65) - a commencé dans les années soixante, sous le gouvernement de Sukuna. Des personnes non détentrices de titre de chef ont commencé à bénéficier d'une position économique et d'un prestige social qui se rapprochaient de ceux des hauts chefs qui détenaient des postes gouvernementaux. Quelques-uns ont occupé des fonctions au même niveau que les chefs dans la bureaucratie et fréquentaient les mêmes clubs ou associations. En 1947, le premier commoner à avoir été nommé au conseil législatif et ensuite au comité des Affaires fidjiennes (Fijian Affairs) est un enseignant de Tailevu. Le même homme a aussi été nommé Roko Tui Tailevu, acquérant ainsi un titre de chef et une supériorité sociale sur les autres chefs. Il n'était pas inhabituel pour un « roturier " d'être désigné Roko Tui (chef de haut rang), après la Deuxième Guerre mondiale (Norton, 1977). L'attribution du titre visait en quelque sorte à réparer ce qui était considéré comme une " erreur " (par exemple ne pas être né au bon endroit) et à rendre la personne concernée plus « fréquentable ». Quoi qu'il en soit, les années cinquante virent une augmentation du nombre des individus ne descendant pas d'une lignée de chefs représentés dans le gouvernement central et dans l'administration fidjienne. Et quelquesuns d'entre eux ont pu commencer des carrières politiques, en tant que leaders d'associations.

Dans la continuité de la mobilité sociale renforcée sous le régime de Rabuka, le pouvoir représenté par les chefs et leurs statuts, leur mana, s'est transformé dans le cadre d'une nouvelle conjoncture socio-économique. C'est dans ce contexte qu'a pu émerger une nouvelle classe de leader économique et politique, considérée comme peu « légitime ». Elle s'illustre notamment par la figure de George Speight, homme d'affaires devenu le leader visible du coup d'État de 2000 (et néanmoins inconnu de la scène politique fidjienne, au moment des faits).

\section{Figure de l'usurpateur et mana illégitime}

Les « intérêts des Fidjiens indigènes " revendiqués par George Speight ont été largement discutés par le discours dominant durant l'année 2000. L'idée que ceux-ci s'élevaient "contre " les intérêts des Indo-Fidjiens représentés par Chaudhry s'est largement répandue dans la population.

En tant que métis ayant étudié aux États-Unis et revendiquant "la cause indigène ", la figure controversée de George Speight exemplifie la problématique liée à la nouvelle indigénéité fidjienne. Son ascendance européenne, par exemple, a suscité passablement de discussions, voire d'indignations du côté des opposants fidjiens au coup d'État. Speight a parfois été qualifié de "descendant de colon blanc " par les médias et le sens commun. Il faut savoir qu’à Fidji, les personnes nées de couples mixtes sont péjorativement appelées half-caste $(\text { kai loma })^{12}$. À travers les médias locaux, spécialement la radio, de nombreux reproches furent adressés au leader

11. Bati signifie littéralement " dents ", ou « l'arête, le bord, le tournant ". Le terme renvoie à un groupe social qui vivait traditionnellement à la frontière de l'espace d'un autre groupe auquel il devait allégeance.

12. Le terme fidjien signifie littéralement une personne " entre-deux ». 
half-caste, dont celui de ne jamais s'exprimer publiquement en langue fidjienne (mais en anglais), de n'avoir pas de nom fidjien (Speight) et de n'avoir jamais vraiment vécu à Fidji (mais plutôt en Australie et aux États-Unis).

La mère de George Speight, fidjienne, est originaire du village de Naivicula dans la province de Tailevu à l'est de Viti Levu. Son père, Sam Speight, est un ancien parlementaire qui avait été inscrit à la naissance dans le groupe de sa mère, sous le nom de Savenaca Tokainavo, en raison de son ascendance considérée officiellement "illégitime ${ }^{13}$ (Robertson et Sutherland, 2000 : 146). Sam n'utilisa le nom de son père, Speight, que lorsqu'il commença à travailler dans les mines d'or de Vatukoula. Un nom européen, durant la période coloniale, permettait d'obtenir un travail mieux rémunéré (Robertson et Sutherland, 2001: 146). Sam Speight reprit le nom de sa mère, Tokainavo, après les coups d'État de 1987, ce qui l'autorisa à remporter un siège représentant les Fidjiens "indigènes " au Parlement en 1992 (Teaiwa, 2001 : 33) ${ }^{14}$.

La référence à l'indigénéité fidjienne est un enjeu pour les individus nés de père ou de mère non fidjiens. Etre inscrit dans le registre fidjien des naissances, Vola ni Kawa ni Bula (vKB), suffit à pouvoir revendiquer son statut d' "indigène ", celui d'habitant originel légitimement possesseur de la terre de ses ancêtres. En ce sens, l'inscription au vKв confere un statut socio-culturel, mais elle constitue aussi une stratégie politique, particulièrement utilisée sous le système électoral antérieur à 1997. Cette tactique est illustrée notamment par la manœuvre de l'ancien parlementaire, Jim Ah Koy, considéré par divers analystes comme le protecteur et mentor de George Speight (Teaiwa, 2001; Robertson et Sutherland, 2001) ${ }^{15}$. D'ascendance chinoise et fidjienne, l'homme d'affaires millionnaire ${ }^{16} \mathrm{Ah}$ Koy a revendiqué sa " fidjianité " pour les élections de 1992, en obtenant l'enregistrement au vKв du côté du yavusa de sa mère. Son inscription lui a permis d'obtenir un siège et de représenter la circonscription électorale de Kadavu au Parlement.

Les nouvelles configurations du mana autochtone sont interdépendantes des revendications identitaires de personnes de la génération de Speight, d'ascendance mixte comme lui. En effet, à la suite de 1987, les descendants d'Européens se sont progressivement détournés de leur identification historique aux privilèges coloniaux pour revendiquer leur statut de part Fijian (Teaiwa, 2001) :
«George Speight prétend représenter les intérêts fidjiens indigènes. En arborant son nom européen, en parlant exclusivement en anglais, en exhibant ses licences universitaires américaines et australiennes pour mana et en portant des vêtements stylés, Monsieur Speight représente certes les intérêts fidjiens indigènes. Mais ses intérêts fidjiens indigènes ne sont, clairement, ni les intérêts fidjiens indigènes de Ratu Mara, ni ceux de feu Dr. Bavadra. » (Teaiwa, 2001 : 33)

\section{De Mara à Bainimarama, la légitimité du mana en mutation}

Indissociable du lieu d'origine et du groupe d'appartenance, la trajectoire politique d'un leader fidjien est imbriquée dans un réseau d'alliances complexes, anciennes et nouvelles, héritées et/ou acquises. Les privilèges sociaux peuvent être le résultat à la fois d'un héritage de droit commun et/ou d'une acquisition de prestige par le niveau d'instruction et/ou le capital économique.

La carrière politique de Ratu Mara trouve sa légitimité dans son double mana: à la fois fidjien par son ascendance et occidental par son instruction. Celles des Premiers ministres qui lui ont succédé trouvent par contre leur légitimité dans d'autres facteurs conjoncturels. En contraste avec le personnage de Ratu Mara, Rabuka, par exemple, a développé sa propre popularité à partir de son image de "self-made man ", converti aussi bien à l'éthique capitaliste que chrétienne. Une éthique par laquelle l'ambition d'un homme d'affaires tel que George Speight a pu se consolider.

Mara, Rabuka, Speight ou Bainimarama incarnent autant de figures du mana « indigène ": le premier construit sur une ascendance dite noble consolidée par une instruction occidentale à Oxford ; l'autre élaboré sur une ascendance « de guerrier » confirmée par une carrière militaire et une ferveur chrétienne; le troisième, d'ascendance mixte, arrivé par l'instruction aux États-Unis et les affaires en Australie ; et le quatrième, Premier ministre depuis 2009, officiellement élu en 2014, fervent démocrate au passé militaire, qui n'a eu de cesse depuis sa présidence intérimaire en 2000, de dénoncer publiquement les dérives de l'ethnonationalisme fidjien.

Le mana de Bainimarama s'est construit sur son combat " pour " la démocratie depuis 2000, plus spécifiquement pour une égalité de droits entre citoyens de Fidji, indépendamment de leur origine ethnique. Originaire de Bau dans l'est de

13. C'est-à-dire qu'il est né d'une union non consacrée par un mariage d'Église. Son père était un fermier européen de Tailevu.

14. Avant la Constitution de 1997, le système électoral fidjien était ethniquement divisé en trois listes : on votait pour des Fidjiens, des Indiens ou des Autres (Generals).

15. Ah Koy avait été suspecté d'avoir financé les coups d'État de Rabuka, en 1987. En 2000, ses liens avec la famille de George Speight ont ravivé la même suspicion.

16. Propriétaire de la firme informatique Datec. 
Fidji, Bainimarama est considéré populairement comme un véritable héros : le Fijis Daily Post l'a consacré "homme de l'année ", à la fin 2000, pour son implication en faveur d'un maintien de la démocratie durant le conflit. Depuis son coup d'État en 2006, il n'a cessé pourtant de repousser les élections "démocratiques" promises, ce qui lui a permis d'asseoir son pouvoir, d'effectuer des changements dans le gouvernement et de mettre en place une nouvelle Constitution (2013). En parallèle de ce combat, Bainimarama a suspendu le grand conseil des chefs en 2007, avant de prononcer son abrogation en 2012. Pour cette occasion, le leader autoproclamé accusa le conseil d'être « devenu politisé au détriment de la poursuite par Fidji d'une citoyenneté commune et égale " (Bainimara, 13 mars 2012). Il serait depuis considéré comme un chef légitime à part entière (Lal, 2012). En juin 2014, Bainimarama a choisi de quitter l'armée pour pouvoir se porter candidat aux élections qu'il remporta en septembre de la même année Ce changement de statut (de militaire à civil) pour "entrer en démocratie " rompt avec le style de leadership d'un Rabuka, qui lui n'a jamais quitté son statut de bati. Fin stratège et/ou démocrate convaincu, Bainimarama instaure assurément une rupture avec le style et la rhétorique des coups précédents. D'un autre côté par contre, son action renforce une continuité avec les leaderships précédents, en regard des répercussions économiques et politiques sur l'archipel. En effet, depuis 1987, des récurrences se sont installées, coup après coup, comme les sanctions internationales qui pénalisent Fidji sur les plans économique et politique, le pourcentage de population indofidjienne qui ne cesse de diminuer pour cause d'émigration successive $(30 \%$ de la population aujourd'hui) ou le taux de pauvreté qui ne cesse de s'accroître (50\% de la population vivait en dessous du seuil de pauvreté en 2012).

\section{La chefferie autrement}

Loin de représenter une structure figée et linéaire (ce qu'elle n’a jamais été), la notion de chefferie doit se comprendre comme une sorte de constellation qui se compose et se recompose de façon subtile, en agençant divers éléments selon des ordres différents et respectivement mouvants, au gré des négociations qui s'actualisent à différentes échelles, locales, nationales, internationales. On l'a vu, isoler l'un de ces éléments pour en faire une caractéristique de la chefferie contre une certaine idée de la démocratie revient à biaiser la compréhension, à évincer la complexité des conjonctures en mouvement.

Depuis plusieurs décennies, la participation de la nation fidjienne à la communauté internationale s'incarne par l'adhésion à plus de quarante organisations internationales économiques, politiques, sociales ou culturelles (dont l'onu, le FMI, Interpol, le Commonwealth, l'oms, le CiO ou le G-77) et des représentations diplomatiques en divers lieux anglophones de la planète dont les États-Unis. Dans ce contexte, les volontés de distinction politique, au plan national, se construisent en interdépendance avec les normes et les valeurs partagées au plan international, dit mondial (Cretton, 2009). Ici, la chefferie s'articule autour d'un éventail de stratégies, lesquelles s'agencent localement parfois en accord, et parfois en désaccord avec l'institutionnalisation de ces normes communes (démocratie), tout en stimulant des manœuvres de distinctions intra, inter et supra-nationales.

Assurément, la chefferie aujourd'hui ne peut plus se comprendre uniquement tel un système traditionnel de chefs aux titres tantôt hérités, tantôt acquis. Les titres et statuts qui s'acquièrent au cours d'une carrière professionnelle, politique, économique, ou militaire (docteur, président, directeur, commandant), participent aussi à la construction du mana indigène. Titres et capitaux hérités et acquis tantôt s'associent et se complètent, tantôt s'affrontent, se côtoient ou s'excluent, selon la façon dont les individus sociaux les activent dans leurs pratiques quotidiennes. À travers les crises politiques et désordres sociaux des deux dernières décennies, une passation de pouvoir semble s'être opérée, des figures coutumières issues de la période coloniale (le noble Ratu Mara) vers des figures militaires plus guerrières (Rabuka, Bainimarama). Des personnalités historiques comme Sitiveni Rabuka, Ratu Mara, George Speight et plus récemment Frank Bainimara, exemplifient chacun à des degrés divers une figure du mana fidjien. Par leur trajectoire, les dirigeants successifs du pays, légitimement élus ou autoproclamés, témoignent du pluralisme inhérent à la figure du chef à Fidji, en illustrant la nouvelle configuration de la classe sociopolitique fidjienne.

\section{Remerciements}

Je remercie Simonne Pauwels pour ses pertinentes suggestions, ainsi que les deux relecteurs anonymes pour leurs remarques et commentaires judicieux.

\section{BIBLIOGRPAHIE}

Arkwright Father N., 2003. Restorative justice in the Solomon islands, in S. Dinnen (ed.), $A$ kind of mending. Restorative justice in the Pacif- 
ic Islands, Canberra, Australian National University, RSPAS, Pandanus Book, pp. 177-194.

Bainimarama Josaia Voreqe, 2012 (13 mars). Remarks by the prime minister on the great council of chiefs (GCC), communiqué de presse (http://www.fiji.gov.fj/Media-Center/Speeches/PM-BAINIMARAMA---REMARKS-ONTHE-GREAT-COUNCIL-OF-CHIEFS-(GCC).aspx, consulté le 15 août 2015).

Clastres Pierre, 1974. La société contre l'État. Recherches d'anthropologie politique, Paris, éditions de Minuit.

Cretton Viviane, 2002a. Chronique d'une ethnographie dans un Parlement assiégé par des rebelles. Le dilemme de l'anthropologue en situation de conflit : participer, mais à quoi ?, Carnets-de-Bord, Revue de Jeunes chercheurs en sciences sociales 3, pp. 78-87.

—, 2002b (décembre). Un coup d'État à Fidji, ou les enjeux d'un terrain bouleversé par l'événement ethnographiques.org 2 (http://www. ethnographiques.org/documents/article/arCretton.html).

—, 2005. Traditional Fijian apology as a political strategy, in Hermann Elfriede et Wolfgang Kempf (eds), Relations in multicultural Fiji: transformations, positionings and articulations. Australia. Special Volume Oceania 75, 4, pp. 403-417.

—, 2007. Négocier le conflit à Fidji. "Cérémonies du pardon" et enjeux du coup d'État de 2000, Paris, l'Harmattan, coll. Mondes océaniens.

—, 2009. L'ambivalente figure de l'indigène universel dans les discours sur la nation Fidjienne, in N. Gagné, Th. Martin et M. Salaün (éds), Autochtonies: vues de France et du Québec, Québec, Les Presses de l'Université Laval, Mondes Autochtones/Dialog, pp. 401-418.

Dinnen Sinclair (ed.), 2003. A kind of mending. Restorative justice in the Pacific Islands, Canberra, Australian National University, RSPAs, Pandanus Book.

Douglas Bronwen, 2003. Les 'États' faibles et les affirmations nationalistes locales: des paradigmes mélanésiens en émergence ?, Ethnologies comparées 6, pp. 1-17 (http://www.alor.univ-motp3.fr/cerce.htm).

Durutalo Alumita, 2003. Informal justice in law and justice reform in the Pacific region, in S. Dinnen (ed.), A kind of mending. Restorative justice in the Pacific Islands, Canberra, Australian National University, RSPAS, Pandanus Book, pp. 165-176.

FIJI'S DAILY POST, 2000 (7 août). Military rejects traditional apology from rebel chiefs, p. 1.
FIJI SUN, 2000 (6 juin). Chiefs want Chaudhry as West PM, p. 3.

—, 2000 (20 juillet). Labasa rebel soldiers claim full support, p. 2.

FIJI TIMES, 2000 (4 juin). Chiefs want west govt. A delegation will inform the Great Council of Chiefs about their intention to form a West Government, p. 1.

-, 2000 (8 juin). West chiefs to decide statuts, p. 1.

France Peter, 1969. The charter of the land: custom and colonization in Fiji, Melbourne, New York, Oxford University Press.

Hau'ofa Epeli, 2008. We are the ocean: selected works, Hawai'i, University of Hawai'i Press.

Hooper Steven J.P., 1982. A study of valuables in the chiefdom of Lau, Fiji, University of Cambridge, Thesis (copie privée de l'auteur).

Jeudy-Ballini Monique, 2004. L'art des échanges. Penser le lien social chez les Sulka, Lausanne, Payot.

KeEsing Roger M., I984. Rethinking mana, Journal of anthropological research 40, 1, pp. 137-156.

Lal Brij V., 1992. Broken Waves. A history of the Fiji Islands in the $20^{\text {th }}$ century, Hawai'i, University of Hawai'i Press.

—, 2000a. Chiefs and thieves and other people beside's: the making of George Speight's coup, The journal of Pacific History 35, 3, pp. 281293.

—, 2000b. Madness in May: George Speight and the unmaking of modern Fiji, in B.V. Lal (ed.), Fiji before the storm. Elections and the politics of development, Canberra, Australian National University, Asia Pacific Press, pp. 175-194.

—, 2012. Editorial: Fij's coup Conundrum, The Roundtable: the commonwealth journal of international affairs 101, 6, pp. 489-497.

Lawson Stephanie, 1991. The failure of democratic politics in Fiji, Oxford, Clarendon Press.

-, 1996. Tradition Versus Democracy in the South Pacific. Fiji. Tonga and Western Samoa, Cambridge, University Press.

LeCKIE Jacqueline, 2002. Return to Nukulau. The troubled waters of ethno-nationalism in Fiji, in E. Kolig et H. Mückler (eds), Politics of indigeneity in the South Pacific, Hamburg and London, Lit Verlag Münster, pp. 119-142.

Mauss Marcel, 1983 (1950). Sociologie et anthropologie, Paris, PUF, Quadrige.

Muller Jean-Claude, 1991. Chefferie, in Pierre Bonte, Michel Izard et al. (eds), Dictionnaire 
de l'ethnologie et de l'anthropologie, Paris, PUF, pp. 138-139.

NayaCakalou R. Rusiate, 1975. Leadership in Fiji, Suva, University of the South Pacific, Institute of Pacific Studies.

NorTON Robert, 1977. Race and politics in Fiji, St-Lucia, University of Queensland Press.

,- 2000 . A paradox of tradition in a modernising society: chiefs and political development in Fiji, in A. Hoope (ed.), Culture and sustainable development in the Pacific, Canberra, Asia Pacific Press, pp. 142-158.

—, 2012. "A pre-eminent Right to political rule": indigenous Fijian power and multi-ethnic nation building, The Roundtable: the commonwealth journal of international affairs 101, 6, pp. 521-535.

Ravuvu Asesela, 1974. Fijians at war 19391945, Suva, University of the South Pacific, Institute of Pacific Studies.

-, 1983. Vaka I Taukei. The Fijian way of life, Suva, University of the South Pacific, Institute of Pacific Studies.

-, 1991. The facade of Democracy. Fijian struggle for political control. 1830-1987, Suva, Reader Publishing House, Procera House.

Robertson Robbie, 2012. Cooking the Goose: Fiji's Coup Culture Contextualised, The Roundtable: the commonwealth journal of international affairs 101, 6, pp. 509-519.

Robertson Robbie and William SutherLand, 2001. Government by the gun. The Unfinished business of Fijis Coup, Australia, Pluto Press.

Routledge David, 1985. Matanitu. The struggle for power in early Fiji, Suva, University of the South Pacific.

Rutz Henry J., 1995. Occupying the Headwaters of tradition: Rhetorical strategies of nation making in Fiji, in J. Foster (ed.), Nation making. Emergent identities in postcolonial Melanesia, Ann Arbor, University of Michigan Press, pp. 71-93.

SAHLINs Marshall, 1961. The segmentary lineage: an organisation of predatory expansion, American Anthropologist 63, 2, pp. 332-345.

-, 1962. Moala. Culture and Nature on a Fijian Island, Ann Arbor, University of Michigan Press.

—, 1968. Poor man, Rich man, Big man, Chief: political types in Melanesia and Polynesia, in A. P. Vayda (ed.), People and Cultures of the Pacific. An anthropoligical reader, New York, Natural History Press, pp. 157-176.
-, 1982. Femmes crues, hommes cuits et autres " grandes choses " des îles Fidji, Le Débat 19, pp. 121-145.

-, 1991. The return of the event again: with reflections on the beginning of the great Fijian war of 1843 to 1855 between the kingdoms of Bau and Rewa, in Biersack A. (ed.), Clio in Oceania. Toward a Historical anthropology, Washington and London, Smithsonian Institution Press, pp. 37-99.

Service Elman Rogers, 1962. Primitive Social Organization, New York, Random House.

Tearwa Teresia, 2001. An Analysis of the current political crisis in Fiji, in B. V. Lal and M. Pretes (eds), Coup. Reflections on the political crisis in Fiji, Canberra, Australian National University, Pandanus Book, pp. 31-34.

TCherkézoff Serge, 2000. Are the Matai 'out of time'? Tradition and democracy: contemporary ambiguities and historical transformations of the concept of chief, in E. Huffer and A. So'o (eds), Governance in Samoa. Pulega I Samoa, Canberra, Australian National University, Asia Pacific Press and Suva, University of the South Pacific, Institute of Pacific Studies, pp. 113-132.

Thomas Nicholas, 1990. Regional Politics, Ethnicity and Custom in Fiji, The Contemporary Pacific 2, 1, pp. 131-146.

-, 1991. The Entangled Objects. Exchange, material culture and colonialism in the Pacific, Cambridge, Massachusetts, Londres, Harvard University Press.

Tuimaleali'ifano Morgan, 2000. Current developments in the Pacific. Veiqati Vaka Viti and the Fiji Islands Elections in 1999, The Journal of Pacific History 35, 3, pp. 253-267.

Tuwere Ilaitia S., 2002. Vanua. Towards a fijian theology of place, Suva, University of the South Pacific, Institute of Pacific Studies.

Waterhouse Joseph, 1997. The king and people of Fiji, Auckland, Pasifikia Press.

White Geoffrey M. and Lamont Lindstrom (eds), 1997. Chiefs today. Traditional Pacific leadership and the postcolonial state, California, Stanford University Press.

Wittersheim Éric, 2002. Qui est chef? La représentation politique de la coutume au Vanuatu, in C. Hamelin et É. Wittersheim (eds), La tradition et l'État. Églises, pouvoirs et politiques culturelles dans le Pacifique, Paris, L'Harmattan, Cahiers du Pacifique Sud Contemporain, pp. 131-160. 